\title{
Development of Novel Thin Film Solar Cells: Design and Numerical Optimisation
}

\author{
Sidi Ould Saad Hamady, ${ }^{1 *}$ Nicolas Fressengeas, ${ }^{1}$ Christyves Chevallier, ${ }^{1}$ Queny Kieffer, ${ }^{1}$ \\ Zainuriah Hassan, ${ }^{2}$ Mohd Anas Ahmad, ${ }^{2}$ Way Foong Lim $^{2}$ and Sha Shiong $\mathrm{Ng}^{2}$ \\ ${ }^{1}$ Université de Lorraine, CentraleSupélec, Laboratoire Matériaux Optiques, \\ Photonique et Systèmes (LMOPS), Metz, France \\ ${ }^{2}$ Institute of Nano Optoelectronics Research and Technology (INOR), \\ Universiti Sains Malaysia, 11800 USM Pulau Pinang, Malaysia \\ ${ }^{*}$ Corresponding author: sidi.hamady@univ-lorraine.fr
}

Published online: 25 December 2019

To cite this article: Hamady, S. O. S. et al. (2019). Development of novel thin film solar cells: Design and numerical optimisation. J. Phys. Sci., 30(Supp. 2), 199-205, https://doi. org/10.21315/jps2019.30.s2.17

To link to this article: https://doi.org/10.21315/jps2019.30.s2.17

\begin{abstract}
The development of cost-effective solar cells requires on the one hand to master the elaboration techniques, and on the other hand, an adequate design to optimise the photovoltaic efficiency. These two research topics are closely linked and their association in the research work is the key in the development of novel thin film solar cells. The design associated with numerical optimisation gives the set of optimal physical and geometrical parameters, taking into account the technological feasibility. This will allow elaboration to target the most efficient structures in order to speed up the final device realisation. In this work, we used a new approach, based on rigorous multivariate mathematical global Bayesian algorithm, to optimise a Schottky based solar cell (SBSC) using InGaN as the absorber. The obtained photovoltaic efficiency is close to the conventional structures efficiency while being less complex to elaborate. In addition, the results have shown that the optimised SBSC structure exhibits high fabrication tolerances.
\end{abstract}

Keywords: Solar cell, photovoltaics, InGaN, Schottky, numerical optimisation

\section{INTRODUCTION}

The optimisation of thin film solar cells involves many physical and technological parameters such as the active layers' composition, doping, thickness, optical parameters and density of defects, etc. The development of numerical optimisation methods using all these parameters are crucial in the design and, in fine, the 
fabrication of cost-effective solar cells. One main prerequisite for these methods is to take into account the solar cell parameters' interdependence. For instance, the optimal composition depends on the doping and the absorber thickness, while the optimal thickness depends on the optical parameters such as the absorption coefficient and the refractive index. To optimise the solar cell efficiency with respect to all these parameters, the so-called parametric analysis is usually used: only one parameter is varied at a time while the other parameters are kept constant. ${ }^{1,2}$ This standard procedure has two main drawbacks. It does not take into account the parameters' interdependence highlighted previously, and secondly, it does not give the absolute optimal efficiency. The new methodology based on a global Bayesian algorithm addressing these drawbacks is presented in the next section.

\section{EXPERIMENTAL}

As an alternative to parametric analysis, we developed a new methodology using nonlinear optimisation algorithms to calculate the set of parameters maximising the efficiency. ${ }^{3}$ This methodology permits to simultaneously optimise a large set of parameters taking automatically into account their interdependence while drastically reduce the computing time when compared to the parametric analysis. The developed code, SoLAr ceLl multivariate OptiMizer (SLALOM), implements this methodology and the including steps. Firstly, one defines a set of crucial parameters to the solar cell operation (e.g., composition, doping concentration and layers thickness). Then, a semiconductor devices simulator is used to perform the numerical evaluation of the solar cell efficiency from its parameters using a mathematical algorithm designed to find the set of values that maximises the efficiency using a numerical iterative procedure. The new implemented optimisation method uses a global Bayesian algorithm. ${ }^{4}$ This global algorithm ensures that the found optimum is absolute and takes less computation time than the previously used methods.

The global Bayesian algorithm implemented in SLALOM is used in this work to optimise a Schottky based solar cell (SBSC) using indium gallium nitride $(\mathrm{InGaN})$ as the absorber. InGaN has the potential to achieve high photovoltaic efficiency since its bandgap can cover the whole solar spectrum, as illustrated in Figure 1, by changing the indium composition. ${ }^{5}$ In addition, this ternary alloy can potentially permit to fabricate cost effective and robust solar cells operating at high temperature and high light intensity, e.g., in concentrator solar cells. The main drawbacks of using this material are the difficulty to grow thin film with high indium composition, the difficulty of p-type doping and the realisation of ohmic contacts. ${ }^{5,6}$ Using the SBSC technology is proposed to address the 
p-doping challenge. The InGaN SBSC structure is shown in Figure 2. The main parameters used in the simulations, taken or extrapolated from experimental data, are summarised in Table 1. The parameters to be optimised for this structure are the Schottky contact workfunction, the indium composition in InGaN, the doping concentration and the layer thickness.

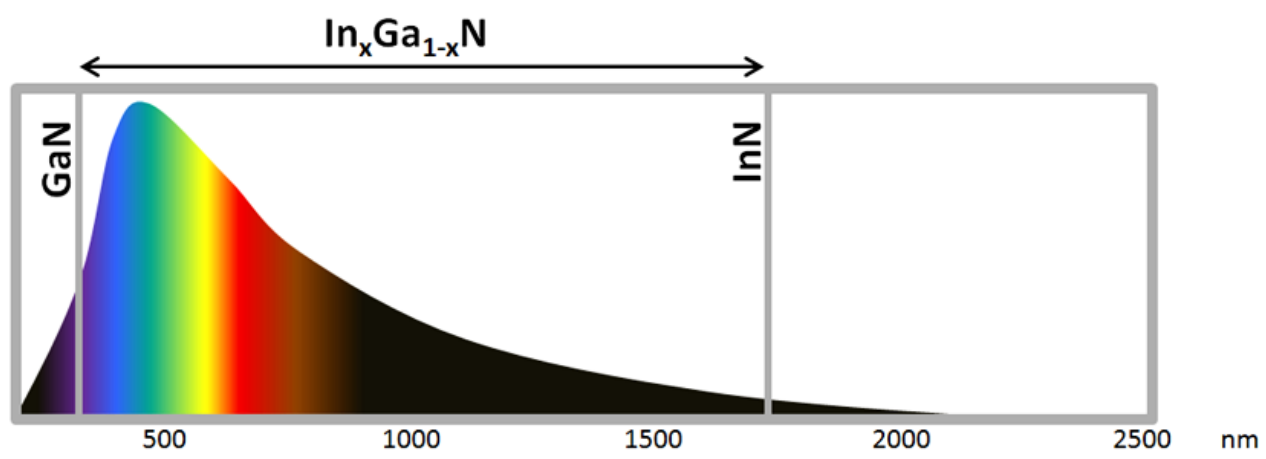

Figure 1: A schematic AM0 solar spectrum showing the matching with the InGaN bandgap with indium composition $x$ varying from $0(\mathrm{GaN})$ to $1(\mathrm{InN})$.

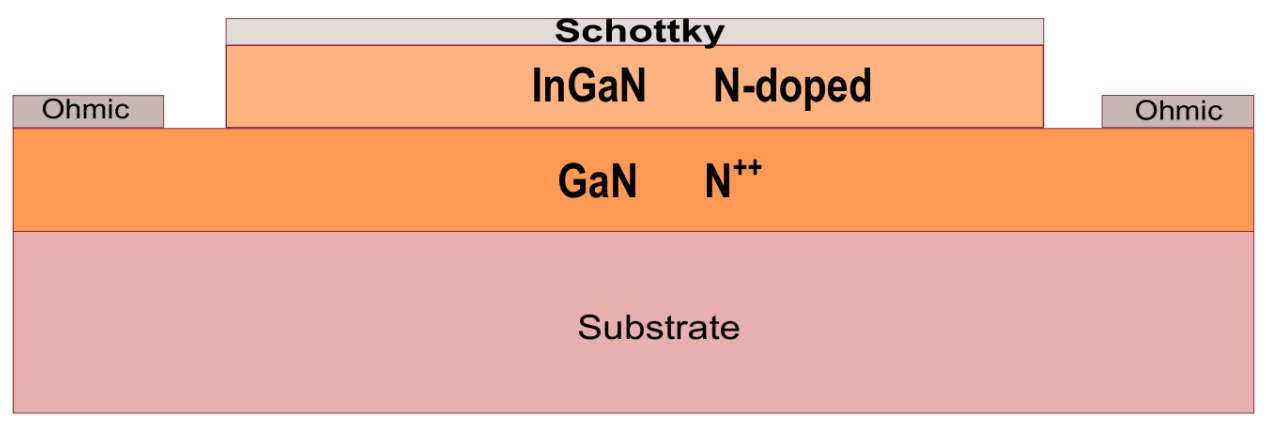

Figure 2: InGaN Schottky based solar cell structure. The InGaN layer is grown on a GaN template substrate on sapphire. The role of the GaN is twofold. It is used as a substrate for the epitaxy growth of the InGaN absorber and to realise the back ohmic contact. It is heavily doped to reduce the ohmic contact resistance. The InGaN doping is $6.5 \times 10^{16} \mathrm{~cm}^{-3}$ with a thickness of $0.86 \mu \mathrm{m}$. The GaN layer doping is about $10^{19} \mathrm{~cm}^{-3}$ and its thickness is about $4 \mu \mathrm{m}$. 
Table 1: The InGaN parameters used in the numerical simulations. Bandgap, affinity, density of states in valence and conduction bands, permittivity, absorption, Shockley-Read-Hall (SRH), Auger and direct recombination data are taken from literature or extrapolated from $\mathrm{GaN}$ and $\mathrm{InN}$ experimental data.

\begin{tabular}{lcc}
\hline Parameters & InN & GaN \\
\hline Bandgap (eV) & 0.7 & 3.42 \\
Affinity (eV) & 5.6 & 4.1 \\
Carrier lifetime (ns) & 1 & 1 \\
Permittivity & 15.3 & 8.9 \\
Density of states in conduction band $\mathrm{N}_{\mathrm{C}}\left(\mathrm{cm}^{-3}\right)$ & $9.1 \times 10^{17}$ & $2.3 \times 10^{18}$ \\
Density of states in valence band $\mathrm{N}_{\mathrm{V}}\left(\mathrm{cm}^{-3}\right)$ & $5.3 \times 10^{19}$ & $4.6 \times 10^{17}$ \\
Absorption & Extracted from experimental data. \\
Mobility & \multicolumn{2}{c}{ Caughey-Thomas model. ${ }^{8}$} \\
Refractive Index & \multicolumn{2}{c}{ Adachi model. ${ }^{7}$} \\
\hline
\end{tabular}

\section{RESULTS AND DISCUSSION}

Figure 3 displays the photovoltaic performances (efficiency, short-circuit current and open-circuit voltage) obtained using the global Bayesian optimisation method. The total computation time, with high precision, is about $2 \mathrm{~h}$ while it takes about $500 \mathrm{~h}$ for the standard parametric analysis, and with lesser precision. The optimised parameters are shown in Table 2. The InGaN SBSC exhibits an optimal efficiency of approximately $22 \%$, very close to the record efficiency obtained for the well-established thin film solar cells. ${ }^{10}$ This obtained result is based on simulations using parameters extracted from experimental data, associated to a rigorous optimisation global Bayesian algorithm. Therefore, we ensure that the obtained efficiency is optimal and not overestimated. This optimal efficiency is obtained for the following set of values: a layer thickness of $0.86 \mu \mathrm{m}$, a doping of $6.5 \times 10^{16} \mathrm{~cm}^{-3}$, a metal workfunction of $6.30 \mathrm{eV}$ and an indium composition of $56 \%$. The indium composition that is necessary for the optimal Schottky solar cell is still challenging. If we decrease the composition down to $30 \%$, the efficiency decreases to around $10 \%$. The simplicity of the SBSC structure, with only one n-doped layer, makes it very cost effective and robust when compared to the standard PN, PIN or multijunction structures.

One aspect, crucial for the development of this technology, is the fabrication tolerance. The tolerance that is allowed on each parameter is the range in which this parameter can vary without decreasing the efficiency by more than $10 \%$ of its maximum. ${ }^{11}$ This latter value is relative. For instance, if the optimised efficiency is $22 \%$, then in the considered parameter's tolerance range the efficiency remains 
between $19.8 \%$ and $22 \%$. Table 2 displays for each optimised parameter the corresponding tolerance range, showing that the proposed InGaN SBSC structure exhibits high fabrication tolerances. For instance, the layer thickness can vary between $0.53 \mu \mathrm{m}$ and $1 \mu \mathrm{m}$ with an efficiency between $19.8 \%$ and its optimal value of $22 \%$. One of the main advantages of decreasing the layer thickness is the possibility to grow epitaxial high-quality layer using less material. In addition, the large tolerance in the doping can permit to use the residual InGaN n-doping without need to intentionally dope the layer.
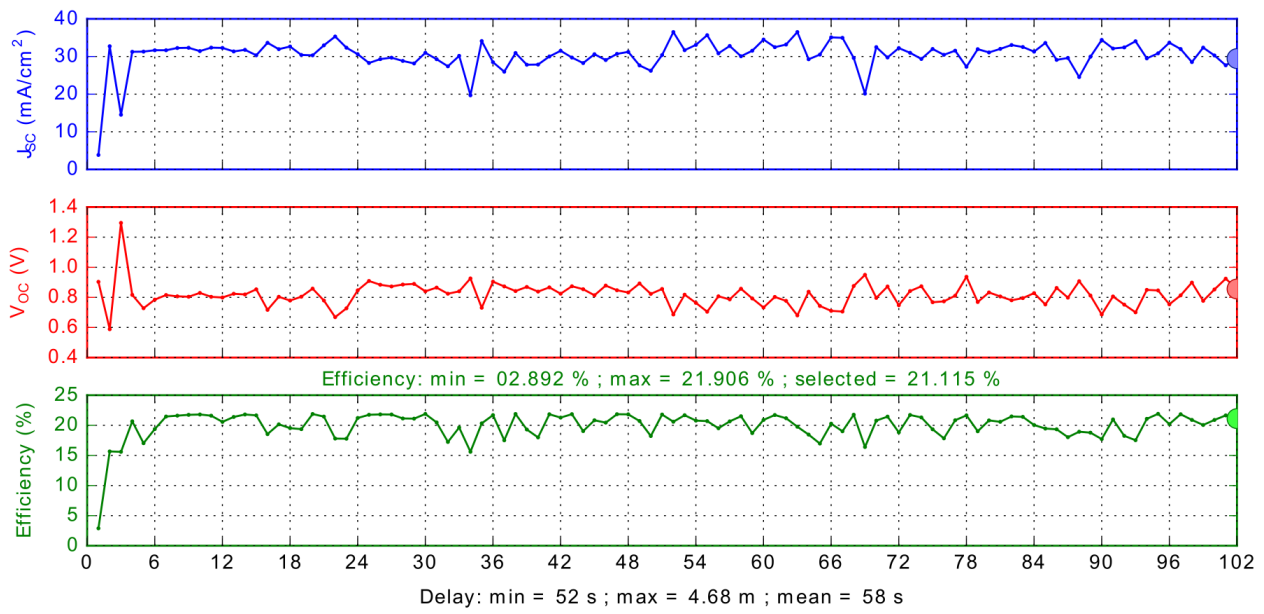

Figure 3: Evolution of the InGaN SBSC photovoltaic performances during the global Bayesian optimisation process. The time (X-axis) is expressed in minutes. $\mathrm{V}_{\mathrm{OC}}$ is the open-circuit voltage and $\mathrm{J}_{\mathrm{SC}}$ is the short-circuit current. The Bayesian algorithm iterates until the desired precision is obtained. In this case, the necessary time for convergence is about $102 \mathrm{~min}$.

Table 2: The optimised InGaN SBSC parameters and the corresponding fabrication tolerance range. The parameters to be optimised for this SBSC structure are the Schottky contact workfunction, the InGaN composition, doping concentration and layer thickness. The optimised efficiency is $22 \%$. In the considered fabrication tolerance range the efficiency remains between $19.8 \%$ and $22 \%$.

\begin{tabular}{lcccc}
\hline & $\begin{array}{c}\text { InGaN thickness } \\
(\mu \mathrm{m})\end{array}$ & $\begin{array}{c}\text { InGaN doping } \\
\left(\mathrm{cm}^{-3}\right)\end{array}$ & $\begin{array}{c}\text { Schottky metal } \\
\text { workfunction }(\mathrm{eV})\end{array}$ & $\begin{array}{c}\text { Indium } \\
\text { composition }\end{array}$ \\
\hline Range & {$[0.10 ; 1.00]$} & {$\left[10^{16} ; 10^{19}\right]$} & {$[5.50 ; 6.30]$} & {$[0.00 ; 1.00]$} \\
Optimum & 0.86 & $6.5 \times 10^{16}$ & 6.30 & 0.56 \\
Tolerance range & {$[0.53 ; 1.00]$} & {$\left[10^{16} ; 3 \times 10^{17}\right]$} & {$[6.15 ; 6.30]$} & {$[0.50 ; 0.72]$} \\
\hline
\end{tabular}


These results pave the way to the actual fabrication of the optimised InGaN SBSC solar cell with two main planned steps. The first step involves the optimisation of the InGaN MOCVD epitaxial growth for high indium composition. ${ }^{12}$ The shortterm objective is to grow an optimised InGaN layer with $20 \%$ of indium and, in mid-term, to increase this composition up to $30 \%$. The second step is the fabrication of the semi-transparent Schottky contact using platinum. The final objective is to obtain the first InGaN SBSC with more than $20 \%$ of indium.

\section{CONCLUSION}

In this paper, a new numerical optimisation method is used to design a Schottky based solar cell using InGaN as the absorber. The global Bayesian algorithm used in this method takes into account the interdependence of the solar cell parameters and ensures the absoluteness of the optimised efficiency. The obtained results, based on using empirical parameters, precise physical models and rigorous optimisation methodology, demonstrates the potential of this technology for cost effective and robust solar cells. The next step, based on this design and numerical optimisation work, is the actual fabrication of the solar cell with the growth of the InGaN absorber and the realisation of the semi-transparent Schottky contact in order to fabricate the first Schottky based solar cell using InGaN with more than $20 \%$ indium.

\section{ACKNOWLEDGEMENTS}

This research was supported by Pôle M4 - Université de Lorraine, Partenariats Hubert Curien (PHC) Hibiscus, INSOL Project and Lorraine Université d'Excellence (LUE) in the framework of the High EfficienCy Thin Film sOlaR cells (HECTOR) research project.

\section{REFERENCES}

1. Zhang, X. et al. (2007). Simulation of $\mathrm{In}_{0.65} \mathrm{Ga}_{0.35} \mathrm{~N}$ single-junction solar cell. J. Phys. D Appl. Phys., 40, 7335-7338, https://doi.org/10.1088/0022-3727\%2F40\% 2F23\%2F013.

2. Feng, S. W. et al. (2010). Theoretical simulations of the effects of the indium content, thickness, and defect density of the $i$-layer on the performance of $p-i-n$ InGaN single homojunction solar cell. J. Appl. Phys., 108, 093118.

3. Hamady, S. O. S. \& Fressengeas, N. (2018). SLALOM: Open-source, portable, and easy-to-use solar cell optimizer. Application to the design of InGaN solar cells. EPJ Photovolt., 9, 13-26. 
4. Locatelli, M. (1997). Bayesian algorithms for one-dimensional global optimization. J. Glob. Opt., 10, 57-76.

5. Fabien, C. A. M. \& Doolittle, W. A. (2014). Guidelines and limitations for the design of high-efficiency InGaN single-junction solar cells. Sol. Energy Mater. Solar Cells, 130, 354-363.

6. Yamamoto, A. et al. (2013). Metal-organic vapor-phase epitaxial growth of InGaN and InAlN for multi-junction tandem solar cells. Mater. Renew. Sustain. Energy, 2, $1-9$.

7. Brown, G. et al. (2010). Finite element simulations of compositionally graded InGaN solar cells. Solar Energy Mater. Solar Cells, 94, 478-483.

8. Schwierz, F. (2005). An electron mobility model for wurtzite GaN. Sol. State Electr., 49, 889-895.

9. Djurisic, A. B. \& Li, E. H. (1999). Modeling the optical constants of hexagonal GaN, InN, and AlN. J. Appl. Phys., 85, 2848-2853.

10. Green, M. A. (2019). Solar cell efficiency tables (version 54). Progr. Photovolt. Res. Appl., 27, 74116.

11. Hamady, S. O. S, Adaine, A. \& Fressengeas, N. (2016). Numerical simulation of InGaN Schottky solar cell. Mater. Sci. Semicond. Process., 41, 219-225.

12. Abud, S. H. et al. (2013). Structural and pptical properties of $\operatorname{In}_{0.27} \mathrm{Ga}_{0.73} \mathrm{~N} / \mathrm{Si}(111)$ film grown using PA-MBE technique. Adv. Mater. Res., 620, 368-372. 